OPEN

SUBJECT AREAS:

APPLIED PHYSICS

SURFACES, INTERFACES AND

THIN FILMS

Received

24 July 2013

Accepted

30 September 2013

Published

31 October 2013

Correspondence and requests for materials should be addressed to C.L.C. (cl.chen@utsa. edu)

* These authors contributed equally to this work.

\section{The Origin of Local Strain in Highly Epitaxial Oxide Thin Films}

\author{
Chunrui Ma ${ }^{1 *}$, Ming Liu'2*, Chonglin Chen ${ }^{1,3}$, Yuan Lin ${ }^{4}$, Yanrong Li ${ }^{4}$, J. S. Horwitz ${ }^{5}$, Jiechao Jiang ${ }^{6}$, \\ E. I. Meletis ${ }^{6}$ \& Qingyu Zhang ${ }^{7}$
}

'Department of Physics and Astronomy, University of Texas at San Antonio, San Antonio, Texas 78249, U.S.A., ${ }^{2}$ Electronic Materials Research Laboratory, Key Laboratory of the Ministry of Education \& International Center for Dielectric Research, Xi'an Jiaotong University, Xi'an 710049, P. R. China, ${ }^{3}$ The Texas Center for Superconductivity, University of Houston, Texas 77204, U.S.A., ${ }^{4}$ State Key Laboratory of Electronic Thin films and Integrated Devices, University of Electronic Science \& Technology of China, Chengdu, Sichuan 610054, P. R. China, ${ }^{5}$ Department of Energy, Basic Energy Sciences, Germantown, MD, 20585, U.S.A., ${ }^{6}$ Materials Science and Engineering, University of Texas at Arlington, Arlington, Texas 76019, U.S.A., ${ }^{7}$ Key Lab of Materials Modification by Laser, Ion, Electron Beams, Dalian University of Technology, Dalian 116024, P. R. China.

The ability to control the microstructures and physical properties of hetero-epitaxial functional oxide thin films and artificial structures is a long-sought goal in functional materials research. Normally, only the lattice misfit between the film and the substrate is considered to govern the physical properties of the epitaxial films. In fact, the mismatch of film unit cell arrangement and the Surface-Step-Terrace (SST) dimension of the substrate, named as "SST residual matching", is another key factor that significantly influence the properties of the epitaxial film. The nature of strong local strain induced from both lattice mismatch and the SST residual matching on ferroelectric $(\mathrm{Ba}, \mathrm{Sr}) \mathrm{TiO}_{3}$ and ferromagnetic $(\mathrm{La}, \mathrm{Ca}) \mathrm{MnO}_{3}$ thin films are systematically investigated and it is demonstrated that this combined effect has a dramatic impact on the physical properties of highly epitaxial oxide thin films. A giant anomalous magnetoresistance effect $\left(\sim 10^{10}\right)$ was achieved from the as-designed vicinal surfaces.

T he lattice misfit between a hetero-epitaxial thin film and the substrate has a very strong influence on the crystal structure, microstructure, and physical properties of the deposited material. There has been a great deal of research on the influence of film strain in hetero-epitaxial films due to the film/substrate lattice misfit. The lattice misfit has been used to enhance the Curie temperature of high temperature superconductors ${ }^{1}$, to alter the Curie temperature of ferromagnetic transition in Colossal Magnetoresistance (CMR) materials $\mathrm{s}^{2-5}$, and to change the dielectric properties of ferroelectric materials ${ }^{6-8}$. In principle, the lattice misfit induced strain energy can be partially or fully released at the interface between the epitaxial film and substrates via formation of edge dislocations that periodically distribute along the interface. This has been directly observed and demonstrated by cross-sectional transmission electron microscopy (X-TEM) microstructural studies ${ }^{9-12}$. However, only a few researches were reported for the investigation of the effect of the mismatch between the film unit cell arrangement and the substrate surface-step-terrace dimensions, named as "SST residual matching", although some investigations have been done on the films grown on various vicinal substrate surfaces ${ }^{13-15}$. It should be noted that there are a large number of surface-step-terraces on single crystal surfaces ${ }^{16,17}$. Normally, a surface-step-terrace will not match an exact number of unit cells or atomic planes in the film, as seen in the Fig. 1. The mismatch of film unit cells/substrate terrace, SST residual matching, will result in an additional strain energy that cannot be released via edge dislocations and will be stored in the hetero-epitaxial films. As the film continues to grow away from the surface, the SST residual matching-induced strain energy will accumulate in the hetero-epitaxial film and can significantly alter its microstructure and thus, its physical and electrical properties. In fact, the effect of surfacestep-terrace on the epitaxial nature of highly epitaxial oxide thin films can be considered in two factors: step height and terrace dimension. The step height can result in the formation of anti-phase domain boundaries (APB), conservative and non-conservative boundaries ${ }^{11,18,19}$. However, the terrace dimension effect is normally neglected in thin film growth. Unlike metal or semiconductor thin films, ionic thin film growth requires a good match in the combination of positive and negative charge balance. Theoretically, when a number of film unit cells fill up along the terrace, a mismatch gap can be generated at the end of the step terrace, which is the "SST residual matching gap" or named as "mismatch gap". Practically, this mismatch gap will not exist at the end of step terrace in an epitaxial film. The last atomic plane of the film will always occupy the atomic position of the terrace end via a 


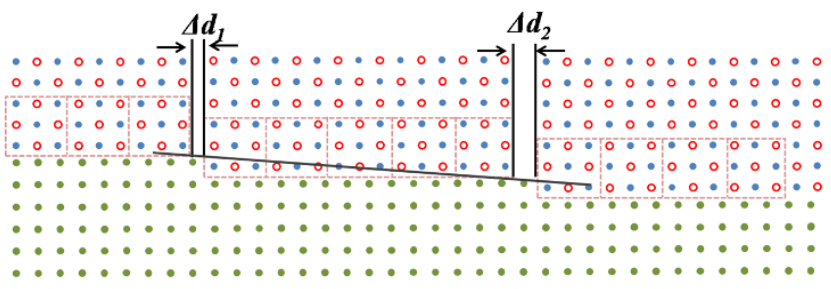

Figure 1 Schematic illustration showing the surface-step-terrace dimension of the substrate can not exactly accommodate integer unit cells or atomic planes of the film.

rearrangement of the local atomic structure. Thus, the atomic position change can significantly increase the growth potential and alter the epitaxial quality.

We have investigated the effects of lattice mismatch on the dielectric properties of $(\mathrm{Ba}, \mathrm{Sr}) \mathrm{TiO}_{3}(\mathrm{BSTO})$ and $2 \% \mathrm{Mn}$ doped $\mathrm{BSTO}$ (Mn:BSTO) films ${ }^{9,20}$. BSTO thin films have been extensively investigated as candidates for development of a new class of tunable microwave signal processing sensors and transducers as well as green energy harvest devices. The films exhibit an electric field dependent dielectric constant and have been used to fabricate tunable capacitors for use in microwave varactors and phase shifters. However, the relatively high loss tangent $(\tan \delta)$ of BSTO films limits their use. It is observed that some materials exhibit a large electric field change in the dielectric constant, but also a high dielectric loss, while other materials exhibit a very small electric field effects, and at the same time a low dielectric loss. The challenge is to optimize the processing of the material such that it exhibits a large electric field induced tuning, while minimizing the dielectric loss ${ }^{10,21,22}$.

CMR effect has been investigated for decade's years ${ }^{23-26}$, since it is a candidate for various electronic device applications, such as the readhead of magnetic memory, especially for the manganite materials and magnetic field sensor. On the other hand, it can provide valuable information to investigate magnetic field induced insulator-metal transition. Here, we report that high epitaxial BSTO and Mn:BSTO thin films on $1^{\circ}, 3^{\circ}$ and $5^{\circ}$ vicinal (001) MgO substrate exhibit different dielectric constant and tunability induced by the SST residual mismatch. Also, the ( $\mathrm{La}, \mathrm{Ca}) \mathrm{MnO}_{3}$ thin film on vicinal (001) $\mathrm{MgO}$ substrate exhibits extremely high magnetoresistance $\left(\sim 10^{10}\right)$, which is about four orders higher than the previous reported record. Thus, we successfully utilize film/substrate interactions to adjust the properties of the material for the desired application.

\section{Results}

To investigate the effect of the residual matching generated from the mismatch between the film unit cells and terrace dimension, we use various miscut (001) $\mathrm{MgO}$ substrates to create different vicinal surfaces for epitaxial growth of ferroelectric BSTO and Mn:BSTO thin films. The various miscut (001) $\mathrm{MgO}$ substrates can provide different surface-step-terrace dimensions on the vicinal surfaces. With miscut angles of $1^{\circ}, 3^{\circ}$, and $5^{\circ}$ on the (001) vicinal MgO surfaces, the average step terrace dimension can be simply estimated to be $12.067 \mathrm{~nm}$, $4.022 \mathrm{~nm}$, and $2.413 \mathrm{~nm}$, respectively. These dimensions correspond the average BSTO unit cells of $30.42,10.17$, and 6.06 on the $1^{\circ}, 3^{\circ}$, and $5^{\circ}$ vicinal surface terraces. Fig. 2 gives dielectric property measurements from the epitaxial Mn:BSTO thin films grown on various miscut (001) MgO vicinal surfaces. Typical dielectric properties of both BSTO and Mn:BSTO thin films on these different miscut surfaces are summarized in Table 1 along with properties of films deposited on normal (001) MgO surface. Here, the tunability of the dielectric constant (i.e. change with an applied electric field) is defined in terms of the gap capacitance with a $0 \mathrm{~V}$ bias, and the gap capacitance with a $40 \mathrm{~V}$ bias, Tunability $(\%)=[\mathrm{C}(0 \mathrm{~V})-\mathrm{C}$ $(40 \mathrm{~V})] / \mathrm{C}(0 \mathrm{~V}) \times 100 \%$.

As seen in Table 1, the dielectric constant and dielectric tunability of films grown on $1^{\circ}$ and $5^{\circ}$ miscut (001) MgO surfaces show very similar properties with the normal (0 degree miscut) substrates. However, both the dielectric constant and tunability for the film grown on $3^{\circ}$ miscut substrate are two-thirds of that for the films grown on $1^{\circ}$ and $5^{\circ}$ miscut (001) MgO surfaces. This dramatic difference in dielectric properties is due to the mismatch between the film unit cells in-plane and the terrace dimensions.

To better understand the effect of step terrace dimensions, X-TEM was employed to investigate the epitaxial quality and interface structures of the BSTO films on the (001) MgO vicinal substrates. Figs. 3 (a)-(c) are X-TEM images showing the epitaxial behavior and (d)-(f) are the interface structures for the BSTO films grown on $1^{\circ}, 3^{\circ}$, and $5^{\circ}$ miscut substrate surfaces, respectively. The insets in Figs. 3 (a)-(c) are the selected area electron diffraction patterns (SAED) taken along the [100] zone of the MgO lattice. All three SAED patterns look similar, indicating that all of the films deposited on the miscut vicinal surfaces have good single crystallinity. The films grown on $1^{\circ}$ and $5^{\circ}$ miscut substrates reveal excellent hetero-epitaxy with very smooth surfaces and very sharp interfaces. Furthermore, edge dislocations are uniformly distributed along their entire interfaces. However, the film grown on the $3^{\circ}$ miscut (001) $\mathrm{MgO}$ substrate is very different. It is observed that the film consists of two layers: a highly epitaxial layer
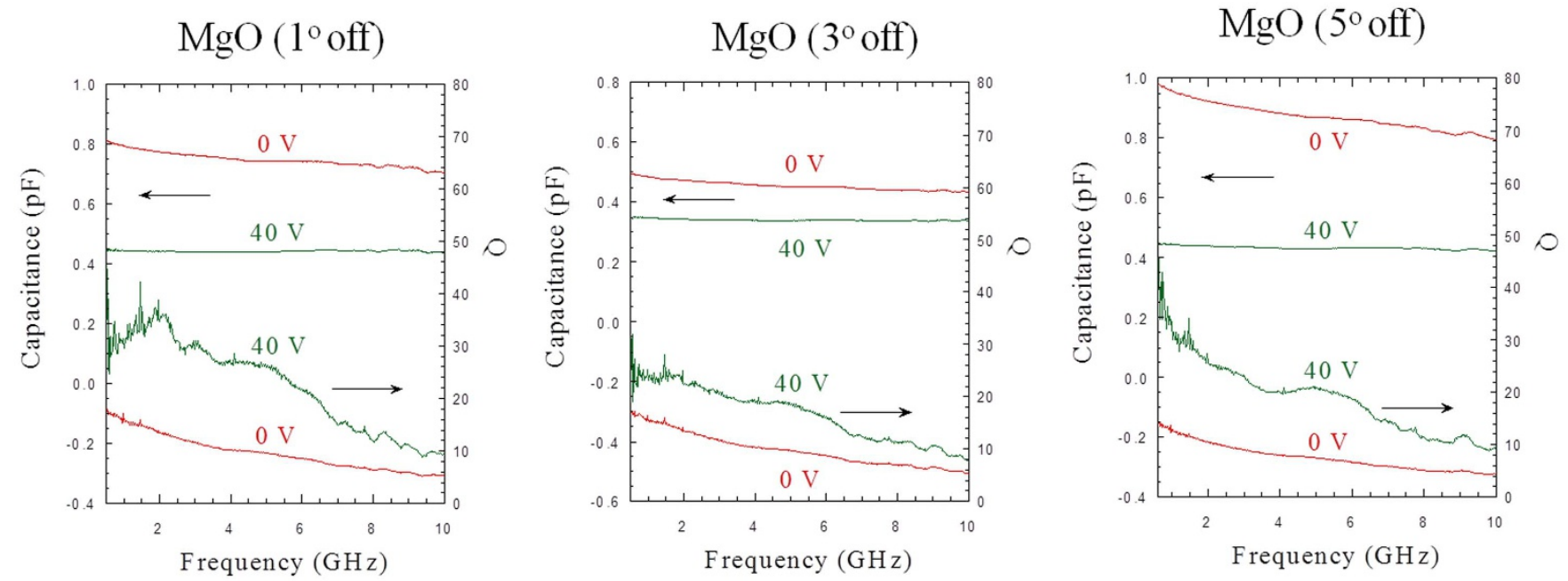

Figure $2 \mid$ Dielectric property measurements showing the effects of surface step terrace induced local strain on the ferroelectric Mn:BSTO thin films grown on $1^{\circ}, 3^{\circ}$, and $5^{\circ}$ miscut substrate surfaces, respectively. 
Table 1 | Dielectric Properties of BSTO and Mn:BSTO thin films on (001) MgO measured at $300 \mathrm{~K}$ and $2 \mathrm{GHz}$

\begin{tabular}{|c|c|c|c|c|c|c|c|}
\hline Sample Types & Residual Match Strain & \multicolumn{3}{|c|}{ Mn:BSTO Films on (001) MgO } & \multicolumn{3}{|c|}{ BSTO Films on (001) $\mathrm{MgO}$} \\
\hline$(001)-0.0^{\circ}$ off & 0 & 1480 & 755 & $49 \%$ & 1068 & 712 & $33 \%$ \\
\hline$(001)-3.0^{\circ}$ off & 0.017 & 1059 & 663 & $37 \%$ & 790 & 598 & $24 \%$ \\
\hline$(001)-5.0^{\circ}$ off & 0.010 & 1655 & 635 & $62 \%$ & 1202 & 790 & $34 \%$ \\
\hline
\end{tabular}

on the miscut $\mathrm{MgO}$ substrate and a top polycrystalline-like layer. However, the electron diffraction pattern (inset in Fig. 3(b), clearly shows that all of the particle-like grains are well aligned along the $c$ axis. Although the interface between the epitaxial film and the $\mathrm{MgO}$ substrate is very sharp, the roughness of the film surface is high (as high as $50 \mathrm{~nm}$ ). In addition, rather than the uniform dissemination, the edge dislocations along the interface are quasi-periodically distributed with a period close to $4.0 \mathrm{~nm}$, as marked by arrows in Fig. 3 (e). These phenomena can be understood by considering the unit cell arrangement on the substrate terraces.

As seen in Fig. 4 (a), the film unit cells are orderly aligned on each terrace of the (001) MgO substrate surface. The surface terminations on the (001) $\mathrm{MgO}$ surface are always the MgO layer although each neighboring $\mathrm{MgO}$ layer has half unit cell height difference. Previous studies of BSTO films on (001) $\mathrm{MgO}$ have indicated that the $\mathrm{TiO}_{2}$ layer is the nucleation layer of the film ${ }^{11}$. Thus, when BSTO grows on the (001) MgO substrate, the hetero-epitaxial BSTO film on each terrace becomes a single domain and the film consists of many domains which are shifted half unit cell along the c-axis if the neighbor terraces are single-step height terraces. The antidomain boundaries are therefore formed at the end of each step terrace. Details can be found in the literature ${ }^{11,27}$. When the BSTO unit cells orderly align along a terrace, the number of BSTO unit cells is determined by the dimension of the terrace. It should be noted that the dimensions of a terrace are not equal to an exact numbers of BSTO unit cells.
Theoretically, the difference of the match between the BSTO unit cells and terrace dimensions will generate a small space gap $\Delta d$ on each terrace, as seen in Fig. 4 (a). However, because of the lattice misfit and the unit cell mismatch on the terrace, each terrace end in the epitaxial growth is always the atomic plane of the film, which results in the unit cells being reconstructed on the terrace, as shown in Fig. 4 (b). This reconstruction results in the formation of local strain domain. The mismatch strain is completely dependent upon the mismatch of the film unit cells and terrace dimension $d$, or simply, the size of the mismatch gap $\Delta d$. The lattice mismatch induced strain can be defined as $\delta=\Delta d / \mathrm{d}$. Unlike the lattice misfit in the film growth, the mismatch-induced strain cannot be released via formation of edge dislocations that generally occur in the lattice misfit.

Thus, for the films grown on vicinal (001) MgO surfaces, the mismatch gaps can be estimated to be $0.42,0.17$, and 0.06 unit cells for the miscut of $1^{\circ}, 3^{\circ}$, and $5^{\circ}$, respectively. For the film on $1^{\circ}$, it can't be inserted the half unit cell of the BSTO film, since it will form no conservative antidomain, Thus, the average local strain distributions of the highly epitaxial BSTO film on the surface step terraces in $1^{\circ}, 3^{\circ}$, and $5^{\circ}$ miscut surfaces can be estimated to be $0.014,0.017$, and 0.010 , respectively. This result indicates that the strain of the BSTO film on the three vicinal substrates is tensile, but the average strain distribution in the $3^{\circ}$ miscut is much larger than the film on the $1^{\circ}$ and $5^{\circ}$ miscut substrate. With film growth, the strain energy will be rapidly
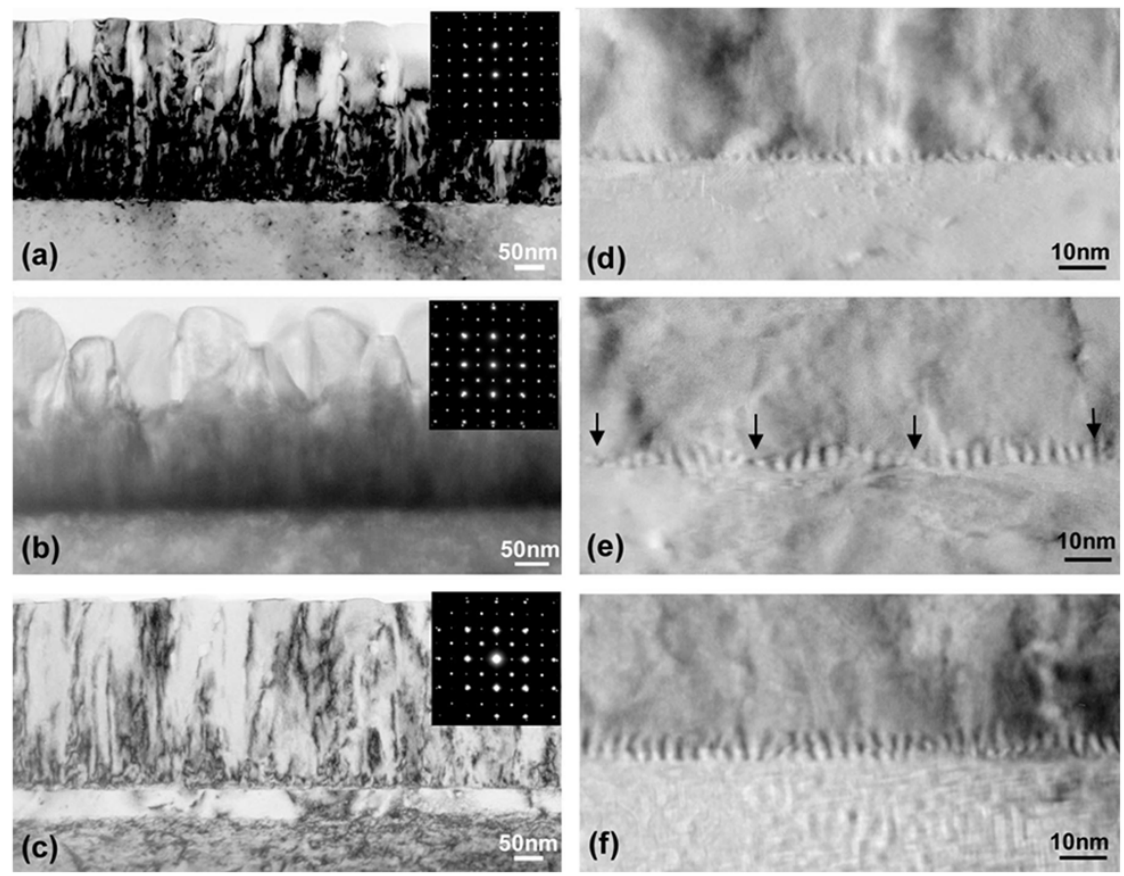

Figure $3 \mid$ (a)-(c) are cross sectional-TEM images showing the epitaxial behavior and (d)-(f) are the interface structures for the BSTO films grown on $1^{\circ}, 3^{\circ}$, and $5^{\circ}$ miscut substrate surfaces, respectively. Edge dislocation distributions for the BSTO films grown on $1^{\circ}$ and $5^{\circ}(\mathrm{d}$ and $\mathrm{f}$ ) are periodic, but it is quasi-periodical on $3^{\circ}(\mathrm{e})^{30}$. The inset of $(\mathrm{a}-\mathrm{c})$ is selection area of electron diffraction patterns (SAED) of the films taken along the [100] zone of the $\mathrm{MgO}$ lattice. 
(a)

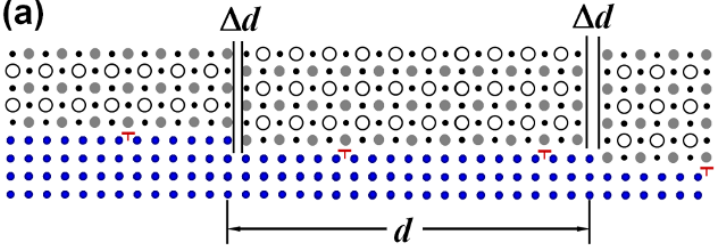

(b)

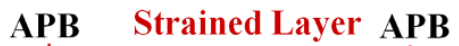

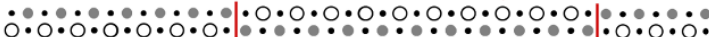

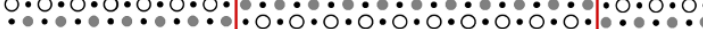

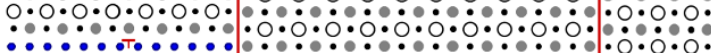

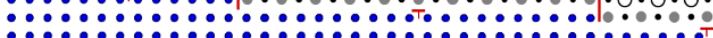

$: \because:$ :

$\bullet \mathrm{Mg}, \mathrm{O} \bullet \mathrm{Ti} \bigcirc \mathrm{Ba}, \mathrm{Sr} \bullet \mathrm{O} T$ Edge dislocation

Figure $4 \mid$ Schematic illustration showing the nature of local strain formation in the highly epitaxial oxide thin films due to the mismatch of film unit cells/substrate surface-step-terrace dimension.

accumulated and stored in the films. When the strain accumulated in the film larger than the critical value for the film, the strain energy must be released with the form which will result in the alteration of the epitaxial behavior by forming the polycrystalline-like particles. This phenomenon is evident in the X-TEM image in the Fig. 3 (b) for the film on the $3^{\circ}$ miscut substrate and can be well understood by growth dynamics under strain.

It is easy to understand the dielectric properties of the present films in terms of the observed structure. The dielectric measurements for the films on $1^{\circ}$ and $5^{\circ}$ miscut substrates display a higher dielectric constant and larger dielectric tunability than the film on the $3^{\circ}$ miscut substrate in the in-planar interdigital measurements. These results are somewhat consistent with the previous studies of the strain effects on BSTO thin films by capacitor measurement ${ }^{27}$ and $(\mathrm{Pb}, \mathrm{Sr}) \mathrm{TiO}_{3}$ (PSTO) thin films on (110) $\mathrm{NdGaO}_{3}$ (NGO) by in-planar interdigitated measurements ${ }^{8}$. In the capacitor measurement, the BSTO films with compressive strain (in-plane) have much larger dielectric constant and higher dielectric tunability (out-plane) than

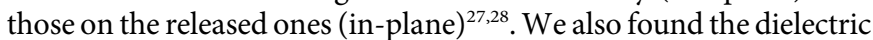
constant and dielectric tunability in the tensile strain direction are 4220 and near $60 \%$ in ferroelectric PSTO thin films on (110) NGO substrates. These values are much greater than those of the films on the compressive strain directions with the dielectric constant value of only 1630 and $33 \%^{8}$. Thus, the films on $1^{\circ}$ and $5^{\circ}$ miscut substrates with tensile strain have large dielectric constant and high dielectric

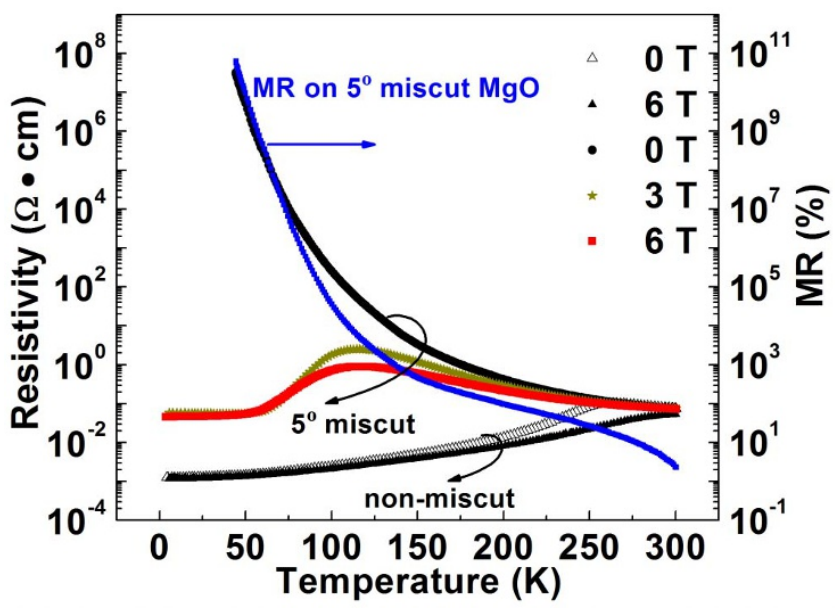

Figure 5 The MR effect measurements showing that the transport properties of the ferromagnetic $(\mathrm{La}, \mathrm{Ca}) \mathrm{MnO}_{3}$ thin films on non-miscut and miscut (001) MgO substrates. tunability in the in-planar interdigitated measurements. Although the film on the $3^{\circ}$ miscut substrate has the largest tensile strain, it has lowest dielectric constant and worst dielectric tenability possibly due to the fact when the strain is too large, it will distorted the crystal structure and generate more defect in the film. On the other hand, a polycrystalline-like particle structure and rough surface will also result in the increase of the dielectric loss and the reduction of the dielectric tunability. The boundary at polycrystalline-like particles and the interface between the highly epitaxial layer and the particle structural layer are usually oxygen deficient, even in the nano twining lamellae structures ${ }^{18}$.

To further verify the local strain formation and its effects of on the physical properties, ferromagnetic $\mathrm{La}_{0.67} \mathrm{Ca}_{0.33} \mathrm{MnO}_{3}$ (LCMO) thin films were epitaxially grown on non-miscut and $5^{\circ}$ miscut vicinal (001) MgO surfaces. Fig. 5 are the transport properties achieved from the non-miscut and $5^{\circ}$ miscut (001) MgO surfaces. The film on the non-miscut $\mathrm{MgO}$ surface shows typical magnetoelectric properties with a clear ferromagnetic transition occurred at $\sim 260 \mathrm{~K}$. However, the film grown on the $5^{\circ}$ miscut vicinal surface reveals an anomalous phenomenon where temperature dependence of resistivity is semiconductor behavior without the application of magnetic field, but under the magnetic field, temperature dependence of the resistivity measurements indicate that a ferromagnetic phase transition occurs at $115 \mathrm{~K}$. Unlike the traditional phase transition for the ferromagnetic LCMO thin films on various substrates and its bulk material in which the transition temperature is increased with the applied magnetic fields, the transition does not change with the applied magnetic fields. Furthermore, a super-large magnetoresistance (MR) effect ratio of $10^{10}$ was achieved from this system, which is about 4 orders higher than the early reported record of MR effect in this system ${ }^{29}$. Details of the mechanisms are under investigation and will be reported later.

\section{Discussion}

This is a systematical study of the local strain effects induced by the film unit cell mismatch with surface step terraces on the physical properties of highly epitaxial oxide thin films. The lattice misfit and unit cell number/substrate terrace dimension mismatch were simultaneously considered. It is shown that this combined effect controls the epitaxial nature and has a dramatic impact on the physical properties of highly epitaxial oxide thin films. This remarkable finding can be utilized as a design tool in the growth of thin film materials for advanced applications.

\section{Methods}

A KrF excimer pulsed laser deposition with a wavelength of $248 \mathrm{~nm}$ was performed to deposit the three different thin films. The $\mathrm{Ba}_{0.6} \mathrm{Sr}_{0.4} \mathrm{TiO}_{3}, 2 \%$ additional $\mathrm{Mn}$ doped $\mathrm{Ba}_{0.6} \mathrm{Sr}_{0.4} \mathrm{TiO}_{3}$ and $\mathrm{La}_{0.67} \mathrm{Ca}_{0.33} \mathrm{MnO}_{3}$ targets were used for the deposition. The BSTO and Mn:BSTO thin films were fabricated on $1^{\circ}, 3^{\circ}$, and $5^{\circ}$ miscut vicinal angles (001) $\mathrm{MgO}$ surfaces. The LCMO thin films were grown on non-miscut and $5^{\circ}$ miscut vicinal (001) MgO surfaces.

The X-TEM was employed to understand the epitaxial quality and interface structures of the BSTO films on (001) MgO vicinal substrates. The dielectric properties (dielectric constant and loss tangent) were determined from interdigitated capacitors which were fabricated on top of the film by standard photolithography. A thin $\mathrm{Cr}$ adhesion layer $(\sim 100 \AA)$ was used for the gold capped silver electrode. The silver was deposited in an e-beam evaporator over the patterned resist. Lift-off was then used to delineate the gap capacitor pattern. Thick silver $(2-5 \mu \mathrm{m})$ was used to reduce the conductor losses at microwave frequencies $(1-20 \mathrm{GHz})$. The transport property measurements of the LCMO thin films were carried out with superconducting quantum interference device.

1. Bozovic, I., Logvenov, G., Belca, I., Narimbetov, B. \& Sveklo, I. Epitaxial strain and superconductivity in $\mathrm{La}_{2}{ }_{\mathrm{x}} \mathrm{Sr}_{\mathrm{x}} \mathrm{CuO}_{4}$ thin films. Phys Rev Lett 89, 107001 (2002).

2. Beach, R. S. et al. Enhanced Curie temperatures and magnetoelastic domains in Dy/Lu superlattices and films. Phys Rev Lett 70, 3502-3505 (1993).

3. Haeni, J. H. et al. Room-temperature ferroelectricity in strained $\mathrm{SrTiO}_{3}$. Nature 430, 758-761 (2004).

4. Tenne, D. A. et al. Probing nanoscale ferroelectricity by ultraviolet Raman spectroscopy. Science 313, 1614-1616 (2006). 
5. Ma, C. R. et al. Thickness effects on the magnetic and electrical transport properties of highly epitaxial $\mathrm{LaBaCo}_{2} \mathrm{O}_{.5+\delta}$ thin films on $\mathrm{MgO}$ substrates. Appl Phys Lett 101, 021602 (2012).

6. Itoh, M. et al. Ferroelectricity induced by oxygen isotope exchange in strontium titanate perovskite. Phys Rev Lett 82, 3540-3543 (1999).

7. Lin, Y. et al. Anisotropic in-plane strains and dielectric properties in $(\mathrm{Pb}, \mathrm{Sr}) \mathrm{TiO}_{3}$ thin films on $\mathrm{NdGaO}_{3}$ substrates. Appl Phys Lett 84, 577-579 (2004).

8. Lin, Y. et al. Epitaxial nature and anisotropic dielectric properties of $(\mathrm{Pb}, \mathrm{Sr}) \mathrm{TiO}_{3}$ thin films on $\mathrm{NdGaO}_{3}$ substrates. Appl Phys Lett 86, 142902 (2005).

9. Gao, H. J. et al. Atomic structure of $\mathrm{Ba}_{0.5} \mathrm{Sr}_{0.5} \mathrm{TiO}_{3}$ thin films on $\mathrm{LaAlO}_{3}$. Appl Phys Lett 75, 2542-2544 (1999).

10. Liu, M. et al. Microwave Dielectric Properties with Optimized Mn-Doped $\mathrm{Ba}_{0.6} \mathrm{Sr}_{0.4} \mathrm{TiO}_{3}$ Highly Epitaxial Thin Films. Cryst Growth Des 10, 4221-4223 (2010).

11. Jiang, J. C., Lin, Y., Chen, C. L., Chu, C. W. \& Meletis, E. I. Microstructures and surface step-induced antiphase boundaries in epitaxial ferroelectric $\mathrm{Ba}_{0.6} \mathrm{Sr}_{0.4} \mathrm{TiO}_{3}$ thin film on MgO. J Appl Phys 91, 3188-3192 (2002).

12. Jiang, J. C., Meletis, E. I., Yuan, Z. \& Chen, C. L. Interface Modulated Structure of Highly Epitaxial (Pb,Sr) $\mathrm{TiO}_{3}$ Thin Films on (001) MgO. Appl. Phys. Lett. 90 , 051904 (2007).

13. McGuigan, L., Barklie, R. C., Sofin, R. G. S., Arora, S. K. \& Shvets, I. V. In-plane magnetic anisotropies in $\mathrm{Fe}_{3} \mathrm{O}_{4}$ films on vicinal $\mathrm{MgO}(100)$. Phys Rev B 77, 174424 (2008).

14. Ma, C. R. et al. Magnetic and Electrical Transport Properties of $\mathrm{LaBaCo}_{2} \mathrm{O}_{5.5+\delta}$ Thin Films on Vicinal (001) $\mathrm{SrTiO}_{3}$ Surfaces. Acs Appl Mater Inter 5, 451-455 (2013).

15. Lu, H. L. et al. Surface-Step-Terrace-Induced Anomalous Transport Properties in Highly Epitaxial $\mathrm{La}_{0.67} \mathrm{Ca}_{0.33} \mathrm{MnO}_{3}$ Thin Films. Acs Appl Mater Inter 2, 2496-2499 (2010).

16. Henrich, V. E. C. P. A. The Surface Science of Metal Oxides. Cambridge University Press (1994).

17. Chen, C. L. \& Tsong, T. T. The Behavior of Ir Atoms and Clusters on Ir Surfaces. Phys. Rev. B 41, 12403 (1990).

18. Jiang, J. C. et al. Two-dimensional Modulated Interfacial structures of Highly Epitaxial Ferromagnetic $(\mathrm{La}, \mathrm{Ca}) \mathrm{MnO}_{3}$ and Ferroelectric $(\mathrm{Pb}, \mathrm{Sr}) \mathrm{TiO}_{3}$ thin films on (001) MgO. JNanoR 3, 59 (2008).

19. Jiang, J. C. et al. Two-dimensional interface structures of epitaxial ( $\mathrm{Ba}, \mathrm{Sr}) \mathrm{TiO}_{3}$ film on miscut (001) MgO. Thin Solid Films 518, 147 (2009).

20. Chen, C. L. et al. Epitaxial ferroelectric $\mathrm{Ba}_{0.5} \mathrm{Sr}_{0.5} \mathrm{TiO}_{3}$ thin films for roomtemperature tunable element applications. Appl Phys Lett 75, 412-414 (1999).

21. Liu, M. et al. Interface Engineered Ferroelectric $\mathrm{BaTiO}_{3} / / \mathrm{SrTiO}_{3}$ Heterostructures with Anomalous Clamped Polarization on Si (100). Integr Ferroelectr 131, 89-94 (2011).

22. Liu, M. et al. Interface Engineered $\mathrm{BaTiO}_{3} / \mathrm{SrTiO}_{3}$ Heterostructures with Optimized High-Frequency Dielectric Properties. Acs Appl Mater Inter 4, 5761-5765 (2012).

23. Dagotto, E., Hotta, T. \& Moreo, A. Colossal magnetoresistant materials: The key role of phase separation. Phys Rep 344, 1-153 (2001).
24. Schiffer, P., Ramirez, A. P., Bao, W. \& Cheong, S. W. Low-Temperature Magnetoresistance and the Magnetic Phase-Diagram of $\mathrm{La}_{1-\mathrm{x}} \mathrm{Ca}_{\mathrm{x}} \mathrm{MnO}_{3}$. Phys Rev Lett 75, 3336-3339 (1995).

25. Millis, A. J., Shraiman, B. I. \& Mueller, R. Dynamic Jahn-Teller effect and colossal magnetoresistance in $\mathrm{La}_{1-\mathrm{x}} \mathrm{Sr}_{\mathrm{x}} \mathrm{MnO}_{3}$. Phys Rev Lett 77, 175-178 (1996).

26. Millis, A. J. Lattice effects in magnetoresistive manganese perovskites. Nature 392, 147-150 (1998)

27. Chen, C. L. et al. Epitaxial growth of dielectric $\mathrm{Ba}_{0.6} \mathrm{Sr}_{0.4} \mathrm{TiO}_{3}$ thin film on $\mathrm{MgO}$ for room temperature microwave phase shifters. Appl Phys Lett 78, 652-654 (2001).

28. Hyun, S. \& Char, K. Effects of strain on the dielectric properties of tunable dielectric $\mathrm{SrTiO}_{3}$ thin films. Appl Phys Lett 79, 254-256 (2001).

29. Xiong, G. C. et al. Giant Magnetoresistance in Epitaxial $\mathrm{Nd}_{0.7} \mathrm{Sr}_{0.3} \mathrm{MnO}_{3-\delta}$ ThinFilms. Appl Phys Lett 66, 1427-1429 (1995).

30. Lin, Y. \& Chen, C. L. Interface effects on highly epitaxial ferroelectric thin films. J Mater Sci 44, 5274-5287 (2009).

\section{Acknowledgements}

This research was partially supported by the National Science Foundation under NSF-NIRT-0709293, the Natural Science Foundation of China under 11329402 and 18110225 , the Department of Energy under DE-FE0003780, and the State of Texas through the Texas Center for Superconductivity at the University of Houston. We would like to thank Dr. HD Wu for the dielectric property measurements of the BSTO films. Also, Dr. Ming Liu and Dr. Chunrui Ma would like to acknowledge the support from the "China Scholarship Council" for the program of national study-abroad project for the postgraduates of high level universities at UTSA.

\section{Author contributions}

C.M. had the magnetic measurements. M.L. had the sample preparation and assisted magnetic measurements. C.C. designed and setup the research and wrote the paper. Y.L. made the Mn:BSTO films and $\mathrm{x}$-ray characterizations. Y.L. assisted manuscript preparation and data analysis. J.H. made the microwave characterizations. J.J. and E.I.M. had the TEM studies and microstructural analysis. Q.Y. assisted the data analysis. All authors discussed the results and commented on the manuscript.

\section{Additional information}

Competing financial interests: The authors declare no competing financial interests.

How to cite this article: Ma, C.R. et al. The Origin of Local Strain in Highly Epitaxial Oxide Thin Films. Sci. Rep. 3, 3092; DOI:10.1038/srep03092 (2013).

This work is licensed under a Creative Commons Attribution-

NonCommercial-NoDerivs 3.0 Unported license. To view a copy of this license, visit http://creativecommons.org/licenses/by-nc-nd/3.0 